\title{
El aerosol refrigerante reduce el dolor de la colocación de catéteres intravenosos
}

\author{
Vapocoolant spray reduces the pain of intravenous cannula placement
}

Hijazi.R. y col .J. BMJ 2009;338:b215

\section{Objetivo}

Evaluar la eficacia, aceptabilidad y seguridad de un aerosol refrigerante para reducir el dolor durante la colocación de catéteres intravenosos en adultos.

\section{Diseño y lugar}

Ensayo clínico aleatorizado controlado y doble ciego, realizado en el departamento de emergencias de un hospital de Melbourne, Australia.

\section{Pacientes e intervención}

Se incluyeron 201 pacientes adultos (promedio de edad 58,2 años) que requirieron la colocación de un catéter intravenoso. Se vaporizó con agua (98 pacientes, grupo control) o refrigerante (103 pacientes, grupo intervención), 15 segundos antes el área de la venopunción, a una distancia de $12 \mathrm{~cm}$ durante 2 segundos.

\section{Medición de resultados principales}

Dolor con la canalización mediante escala analógica visual que va de 0 a $100 \mathrm{~mm}$, y molestia con el aerosol.

\section{Resultados Principales}

El dolor se redujo significativamente en el grupo intervención (Tabla 1). Fue necesario tratar con refrigerantes a 4 pacientes (IC95\% 3-7) para evitar dolor significativo en una persona.

Tabla 1. Resultados principales en el grupo control e intervención.

\begin{tabular}{c|c|c|c} 
& $\begin{array}{c}\text { Control } \\
(\mathbf{N = 9 8})\end{array}$ & $\begin{array}{c}\text { Intervención } \\
(\mathbf{N = 1 0 3 )}\end{array}$ & $\mathbf{P}$ \\
\hline Mediana de dolor & $36(19-51)^{\star}$ & $12(5-40)^{\star}$ & $<0,001$ \\
\hline Dolor mayor de 30mm & $59(60 \%)$ & $33(32 \%)$ & $<0,001$ \\
\hline Mediana de molestia por el aerosol & $0(0-0)^{\star}$ & $0(0-11)^{\star}$ & $<0,001$ \\
\hline Éxito de la colocación del catéter & $73(75 \%)$ & $83(81 \%)$ & 0,390 \\
\hline Reelección del uso del aerosol & $34(39 \%)$ & $62(62 \%)$ & 0,002 \\
\hline Correcto uso del aerosol & $68(69 \%)$ & $56(54 \%)$ & 0,001 \\
\hline
\end{tabular}

${ }^{*}$ Rango intercuartilo

\section{Conclusiones}

El aerosol refrigerante es efectivo, aceptable y seguro en la reducción del dolor por colocación de catéteres intravenosos en pacientes adultos en el departamento de emergencias.

\section{Comentario}

La inserción de catéteres venosos periféricos es un procedimiento común en los servicios de emergencias, constituyendo una experiencia incómoda y dolorosa para muchos pacientes. Los agentes tópicos analgésicos y anestésicos se utilizan con escasa frecuencia en estos ámbitos, ya que es necesario disponer de tiempo para que comiencen a actuar y pueden asociarse a demoras inaceptables en entornos de emergencia. Este ensayo controlado, aleatorizado y doble ciego, evaluó la reducción del dolor con el uso de un aerosol refrigerante aplicado previamente a la colocación de un catéter periférico. Si bien el ensayo clínico constituye el mejor diseño para resolver cuestiones de terapéutica, ${ }^{1}$ este estudio presenta algunas limitaciones, como por ejemplo la posibilidad de que la muestra no haya sido lo suficientemente grande como para discriminar diferencias entre los distintos calibres de los catéteres (agujas de mayor tamaño podrían provocan mayor dolor). Otro aspecto a tener en cuenta es la presencia de los denominados factores de confusión, como el umbral de la ansiedad presente en los pacientes frente al tamaño de la aguja y la sensación de dolor, aunque la aleatorización debería haber podido distribuir estos factores de manera más o menos balanceada en ambos grupos. El resultado de este estudio se suma a la evidencia previa, muchas veces contradictoria, ya que algunos estudios no encontraron diferencias estadísticamente significativas ${ }^{2,3,4}$ mientras que otros sí lo hicieron ${ }^{5,6,7}$. Sin embargo los estudios negativos no fueron doble ciego, difirieron en la técnica de aplicación o tuvieron muestras pequeñas.

\section{Conclusiones de la comentadora}

Si bien este estudio proporciona evidencia a favor del uso de aerosoles refrigerantes, son necesarios más estudios con un buen tamaño muestral, probablemente un metanálisis, que confirmen los resultados antes de recomendar su uso rutinario.

Mariana Patricia Fruncillo [ Servicio de Medicina Familiar y Comunitaria. Hospital Italiano de Buenos Airesr ]

Fruncillo M. El aerosol refrigerante reduce el dolor de la colocación de catéteres intravenosos. Evid. actual. práct. ambul; $13(2)$ : 49 , Abril-Jun 2010. Comentado de Hijazi R et al. Effect of topical alkane vapocoolant spray on pain with intravenous cannulation in patients in emergency departments: randomised double blind placebo controlled trial. BMJ 2009;338:b215. doi:10.1136/bmj.b215. PMID: 19208703.

\section{Referencias}

1. Polit DF, Hungler BP. Investigación científica en ciencias de la Salud.6 $6^{\text {a }}$ Ed Mèxico Mc Graw Hill

2. Hartstein BH,Barry JD. Mitigation of pain Turing intravenous catheter placement using a topical skin coolant in the emergency departamento.Emerg Med J 2008;25:257-61. 3. Costello M, Ramundo M, Christopher NC, Powell KR. Ethyl vinyl chloride vapocoolant spray mails to decrease pain associated with intravenous cannulation in children. Clin Pediatr (Phila) 2006;45:628-32.

4. Biro P, Meier T, Cummins AS. Comparison of topical anaesthesia methods for venous cannulation in adults. Eur J Pain 1997;1:37-42.

5. Selby IR, Bowles BJ. Analgesia for venous cannulation: a comparison of EMLA (5 minutes application), lignocaine, ethyl chloride, and nothing. J R SocMed 1995;88:264-7. 6. Armstrong P, Young C,McKeown D. Ethyl chloride and venepuncture pain: a comparison with intradermal lidocaine. Can J Anaesth 1990;37:656-8.

7. Farion KJ, Splinter KL, Newhook K, Gaboury I, Splinter WM. The effect of vapocoolant spray on pain due to intravenous cannulation in children: a randomized controlled trial. CMAJ. 2008 Jul 1;179(1):31-6. 